\title{
Transcortical approaches to large intraventricular tumors: a prospective case series of 20 patients
}

\author{
Mohamed AbdAllah Elkallaf ${ }^{1,2^{*}}$, Waleed Elsaadany ${ }^{1}$, Wael Mohamed Mohamed Moussa ${ }^{1}$ and \\ Ahmed Abdel Aziz Fayed ${ }^{1}$
}

\begin{abstract}
Background: Large intraventricular tumors (IVTs) impose technical and surgical challenges, due to their enormous sizes, mass effect, vast extensions, and vascularity.

Objective: The authors aim at presenting their results, clinical outcomes, and the surgical strategies in the management of large IVTs through transcortical approaches.

Methods: A prospective trial was conducted at the Main Hospital of Alexandria University, Egypt, between August 2018 and October 2020 on 20 patients harboring IVTs larger than $5 \mathrm{~cm}$ or bilaterally represented. The variables evaluated included the extent of resection, postoperative neurological deficits, blood loss, surgical approaches, intraoperative challenges, complications, adjuvant therapies, Glasgow Outcome Scale, hydrocephalus, and cerebrospinal fluid (CSF) diversion.
\end{abstract}

Results: The study included 20 cases (9 males and 11 females). Mean age at diagnosis was 16.1 years (range 1-45). Mean follow-up was 12 months (range 9-26). Primary tumor locations were ventricular body, atrium, temporal horn, and frontal horn in 11,5,3, and 1 cases, respectively. Main pathologies were central neurocytomas (7 patients/35\%), ependymomas (3 patients/15\%), and subependymal giant cell astrocytoma (SEGA) (3 patients/15\%). Excision was gross total $(n=19)$ and near-total $(n=1)$. Significant intraoperative bleeding $(n=6)$. Postoperative minor intraventricular hemorrhage $(n=6)$, subdural collections $(n=8)$, hydrocephalus $(n=9)$, memory affection $(n=4)$, and motor deficits $(n=4)$. Postoperative Glasgow Outcome Scale of 5/5 $(n=20)$, no mortalities were recorded. preoperative seizures $(n=7)$, new onset postoperative seizures $(n=2)$.

Conclusion: Surgical approaches to large tumors of the lateral ventricles should be tailored to the variability of tumor locations, dimensions, extensions, and the individual morbid anatomy, in addition to the surgeon's experience and preferences. Trans-cortical approaches can provide safe surgical corridors to tackle these challenging subtypes of IVTs with good clinical outcomes, tumor resectability rates, and seizure control.

Keywords: Bilateral tumors, Brain tumors, Central neurocytoma, Intraventricular, Large tumors, Lateral ventricle, Transcortical, Ventricular tumors

\footnotetext{
* Correspondence: dr.m.elkallaf@gmail.com

'Department of Neurosurgery, Faculty of Medicine, Alexandria University, Al Sultan Hussain St., Al Azarita, Alexandria, Egypt

${ }^{2}$ Department of Neurosurgery, Faculty of Medicine, Helwan University, Helwan, Egypt
}

\section{Springer Open}

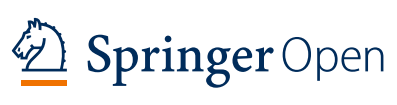

(c) The Author(s). 2021 Open Access This article is licensed under a Creative Commons Attribution 4.0 International License, which permits use, sharing, adaptation, distribution and reproduction in any medium or format, as long as you give appropriate credit to the original author(s) and the source, provide a link to the Creative Commons licence, and indicate if changes were made. The images or other third party material in this article are included in the article's Creative Commons licence, unless indicated otherwise in a credit line to the material. If material is not included in the article's Creative Commons licence and your intended use is not permitted by statutory regulation or exceeds the permitted use, you will need to obtain permission directly from the copyright holder. To view a copy of this licence, visit http://creativecommons.org/licenses/by/4.0/. 


\section{Introduction}

Shaw's report in 1854 was one of the earliest reports on intraventricular tumors (IVTs) in the history of neurosurgery [1]. In many studies, tumors of the lateral ventricle constitute about 0.7 to $3 \%$ of all brain tumors and have a high predilection towards pediatric population [2-6]. These tumors are considered primarily IVTs if they arise from the ventricular lining and are considered secondarily intraventricular if they arise from the brain parenchyma outside the ventricles, but express exophytic extension of more than two-thirds of their masses inside the cavity of the lateral ventricles.

The cerebral ventricular system is complex and encompasses unique anatomic challenges to neurosurgeons who deal with IVTs. This is due to the vast supratentorial and infratentorial extensions of the ventricular system in almost all skull potential spaces and fossae [7, 8]. Therefore, the clinical presentations of IVTs vary depending on the specific location, histopathology, and tumor extensions. Not infrequently do IVTs acquire large sizes before medical attention is drawn to them, and thus diagnosis may be delayed in many cases [9]. Exceedingly large or bilaterally occurring IVTs are rare subtypes of IVTs.

Despite the significant advances in neurosurgical equipment and technology, surgery of large or bilateral IVTs still represents significant challenges due to the deep location of these tumors, the potential disruption of cerebral cortex, eloquent areas, or the corpus callosum while seeking access to these tumors, and the necessity of dealing with deep venous and choroidal arterial structures in many circumstances. Different open and endoscopic surgical approaches have been described for intraventricular tumors, those included transcortical, trans-callosal, and supracerebellar infratentorial approaches. Trans-cortical approaches are considered efficient in excising a wide variety of intraventricular tumors and other pathologies [10-15].

The authors aim at presenting their results and surgical strategies in the management of these challenging tumors through transcortical approaches, together with clinical outcomes.

\section{Methods}

A prospective trial was conducted at the Main Hospital of Alexandria University of Egypt between August 2018 and October 2020, and included 20 consecutive patients with IVTs which had dimensions greater than $5 \mathrm{~cm}$ or had bilateral ventricular extension or representation, and were operated through transcortical approaches. The approval of the Research Ethics Committee (REC) of the Faculty of Medicine, Alexandria University, Egypt, was granted for this study. Patients with prior surgeries or adjuvant therapies were excluded from the study.
All patients had complete preoperative clinical assessment, together with full basic laboratory investigations. The higher functions of the brain were assessed as a part of the comprehensive neurological examination which also addressed the patients' average IQ, understanding, cooperation, memory, attention, spatial orientation, calculation abstract thinking, in addition to detailed history taking including history from the immediate contacts and relatives. Combining these with the Glasgow Outcome Scale (GOS) findings enabled clinical outcome assessment in the studied patients. Computed tomography (CT) and magnetic resonance imaging (MRI) were preoperatively performed as well for all patients to assess the sizes and extensions of the tumors, the patterns of enhancement, and presence of calcifications; the data that guided the main surgical strategy.

In terms of description of tumor locations of the studied IVTs, tumor locations were divided into 2 categories, detailed as follows: (1) the primary main tumor location which describes the ventricular compartment harboring the major bulk of the tumor. (2) The location of the tumor extension or bilateral representation which describe the ventricular compartment harboring the main tumor extension or bilateral representation outside the primary tumor location.

If the tumor arose from one ventricular location with no radiological signs of tumor extensions into other ventricular compartments or tumor bilaterality, the primary main location pattern was then described as "proper."

Regarding the transcortical surgical approaches utilized, the choice of the surgical trajectory was made according to the tumor's main anatomic location, laterality, and extensions, in addition to the preference and the skills of the operating surgeon(s).

Preoperative planning included decision-making as regards the shortest trajectory to the tumor, the location and size of craniotomy, and the suitable length of corticectomy. Locating the vascular tumor pedicle was a constant objective during surgery of the studies cases.

The extent of tumor resection was considered to be gross total resection (GTR) when no tumor residues could be detected intraoperatively or in post-operative MRI, near total resection (NTR) when a residual less than $1 \mathrm{~cm}^{3}$, and subtotal resection (STR) when a residual more than $1 \mathrm{~cm}^{3}$.

Postoperative MRI was planned to be performed within the 48 postoperative hours, then every 3 months in the first 1 year of follow-up, and every 6 months thereafter, unless there was tumor progression or recurrence.

Demographic, clinical, pathologic, and outcome data were assessed prospectively. Descriptive summaries of categorical and ordinal data were presented as numbers and percentages. Age and follow-up periods were 
described in means and ranges. Glasgow Outcome Scale was utilized to assess the clinical outcome postoperatively at 3,6 , and 9 months.

\section{Results}

\section{Patients' demographics}

Twenty patients were included in the study. The mean age at diagnosis was 16.1 years (range 1-45 years). Mean follow-up period was 12 months (range 9-26 months). The chief complaints included manifestations of elevated intracranial pressure (ICP) such as headache, nausea and vomiting in 20 patients, visual deficits in 12 patients, and seizures in 7 patients. Table 1 illustrates the clinical presentations of the studied cases.

\section{Imaging criteria}

Given that the current study deals with very large IVTs possessing various tumor extensions, the tumoral locations were studied and classified into primary main tumor locations and tumor extensions.

The body of the lateral ventricle was found to host the primary location of 11 tumors (55\%) (Fig. 1). Five of which were in the body proper with no extensions. The other six body tumors had various extensions (Fig. 2). The main patterns of anatomical tumor extensions included one biventricular representation, five huge body tumors with bilateral extensions, and three huge atrial tumors with bilateral extensions. Table 2 summarizes the patterns of the primary locations and the extensions of the 20 tumors according to their different anatomic locations within the lateral ventricles. Table 3 summarizes the preoperative imaging criteria of the studied patients.

\section{Surgical approaches}

All tumor excision surgeries were performed through transcortical approaches. The choice of the surgical trajectories depended mainly on the tumor location and extensions, in addition to the personal experience of the operating neurosurgeon(s).

The frontal transcortical approach through the middle frontal gyrus was utilized in 12 cases whose tumors were located in the ventricular body with frontal extensions, ventricular body proper, or bilateral frontal horns (Figs. 3

Table 1 The clinical presentations of large intraventricular tumors

\begin{tabular}{lll}
\hline Preoperative symptoms & No. & $\%$ \\
\hline Headache, nausea, and vomiting (increased ICP) & 20 & 100.0 \\
Visual deficits & 12 & 60.0 \\
Seizures & 7 & 35 \\
Mental, behavioral, or cognitive deficits & 1 & 5 \\
\hline
\end{tabular}

ICP intracranial pressure and 4). While the posterior parietal trans-cortical approach was utilized in 5 complex cases which were primarily located in the atrium, or as extension into the occipital horns or into the atrium from other ventricular compartments. The middle temporal gyrus approach was utilized in three patients with temporal horn IVTs.

It was found that early control of tumor vascularity through tumor pedicle coagulation had a particular impact of the amount of intraoperative bleeding, the easiness of surgical excision, and the duration of surgery.

It is, however, not easy in each case to locate the tumor pedicle from the start. Some tumors' pedicles are embedded on the deep tumor surfaces. Finding and following the potential planes between the tumor and the ventricular walls could help in locating the deep tumor pedicle before directly attacking the main bulk of the tumor. This technique could be performed under the surgical microscope or with the endoscope if the tumor size and surgical planes allow its introduction, and is especially helpful in choroid plexus tumors. Careful assessment of preoperative MRI is mandatory in selecting which plane to follow to reach the tumor's pedicle.

\section{Average blood loss}

Intraoperative complications included significant bleeding of vascular tumors in 6 cases. Bleeding was controllable in all cases with routine measures of hemostasis. Intraoperative and postoperative intravenous fluid therapy was performed utilizing adequate blood components and intravenous fluids. The range of bleeding depended on the extent of tumoral vascularity and the presence of pathological blood vessels, together with the control of tumor vascular pedicle, if feasible. Intraoperative bleeding ranged from 300 to $2000 \mathrm{cc}$.

\section{Extent of resection and histopathological findings}

Gross total resection was achieved in 19 cases and near total resection was achieved in 1 case. Regarding the most common histopathological diagnoses, 7 patients had central neurocytomas (35\%), 3 had ependymomas (15\%), and 3 had SEGA (15\%). Table 4 shows the different histopathological diagnoses of the studied cases.

\section{CSF diversion}

Sixteen patients presented preoperatively with hydrocephalic changes. In nine cases, there had been small postoperative intraventricular bleeds, which were closely observed for signs of elevated ICP, along with serial CT brain scans as indicated. Eventually, all these minor intraventricular bleeds were conservatively managed.

Insertion of external ventricular drains (EVDs) at the end of surgery was resorted to in selected cases when it deemed necessary according to the final microsurgical assessment of the surgical bed. The EVD is left in place 


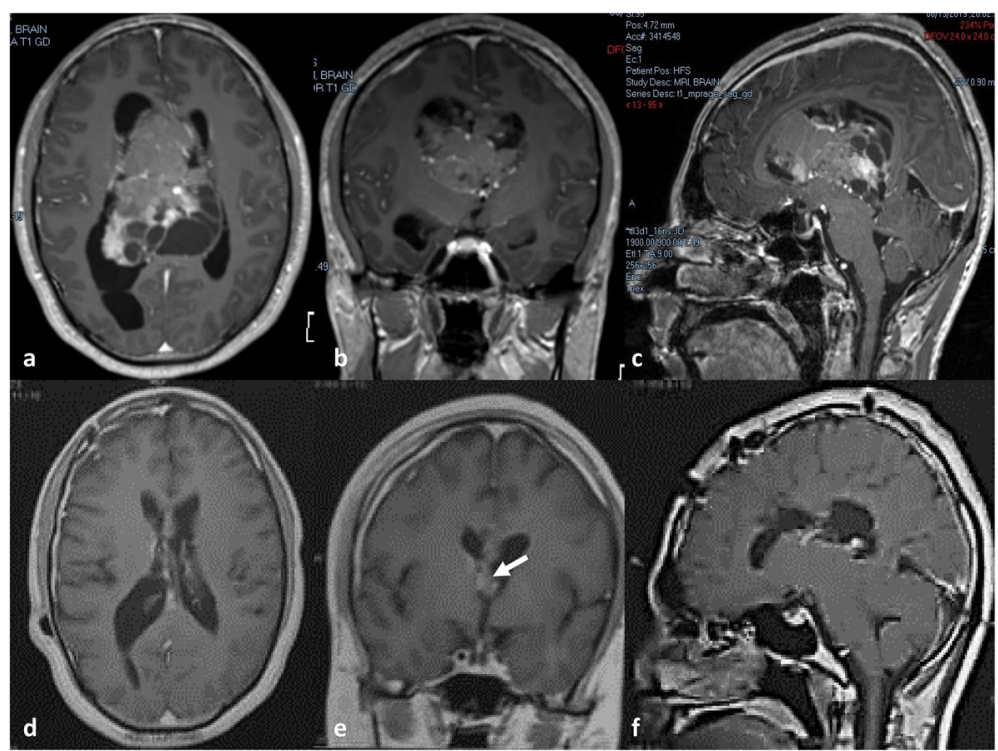

Fig. 1 A large intraventricular central neurocytoma in a 25-year-old male patient, who presented with headaches and blurring of vision. a-c The preoperative axial, coronal, and sagittal contrast-enhanced brain MRI study. $\mathbf{d}-\mathbf{f}$ The postoperative axial, coronal, and sagittal contrast-enhanced brain MRI study. Near-total excision of the tumor was achieved following a right frontal trans-cortical approach through the right middle frontal gyrus, leaving a small septal residue (white arrow)

at $+5 \mathrm{~cm}$ water level until CSF becomes clear, usually by the third post-operative day. During which the daily amount of CSF collected in the EVD reservoir is monitored and recorded. Following this, attempts at gradual weaning from the EVD are exerted with gradual increments of the draining pressure from +5 to $+20 \mathrm{~cm}$ water while monitoring the clinical condition of the patient and the amounts of CSF collected daily, in addition to obtaining computed tomography of the brain upon clinical indications and $24 \mathrm{~h}$ after complete closure of the EVD. If the patient shows dependency on the EVD, ventriculo-peritoneal shunt insertion is warranted. Otherwise, the EVD was usually removed about the fifth post-operative day after a successful process of gradual weaning from the EVD.

In this context, postoperative external ventricular drains (EVDs) were utilized in 12 patients; 7 of which needed permanent ventriculo-peritoneal shunting later. In the other 8 patients, no postoperative EVDs were inserted. Fortunately, 6 out of these 8 patients did not need further forms of CSF diversion. While 1 patient developed a subdural hygroma for which a subduroperitoneal shunt had to be inserted; and the eighth patient developed symptomatic hydrocephalic changes which mandated the insertion of a ventriculo-peritoneal shunt.

\section{Postoperative morbidities}

Postoperative complications included minor intraventricular bleeds in nine patients, extradural hematoma in one case that mandated surgical evacuation, hydrocephalus in nine cases, moderate brain edema in 1 case, subdural hygroma in 7 cases; of which only one needed subdural-peritoneal CSF shunt, transient mild hemiparesis in four cases, in addition to transient short-term memory deficits in four cases, and dysphasia in 1 case. Table 5 shows a summary of postoperative complications and morbidities.

\section{Outcome}

All 20 cases showed GOS of 5 at 3, 6, and 9 months, postoperatively, along with no recorded mortalities.

\section{Adjuvant therapies}

Six cases received adjuvant therapy. On the one hand, two patients with anaplastic ependymomas, two patients with choroid plexus carcinoma, and one patient with atypical choroid plexus papilloma received radiotherapy and chemotherapy. On the other hand, one case with anaplastic meningioma received adjuvant radiotherapy only.

\section{Discussion}

Lateral ventricular tumors are mainly accessed through transcortical and transcallosal interhemispheric approaches. However, various factors have to be considered such as the surgeon's preferences, tumoral location, neurovascular morbid anatomy, and the patient's premorbid conditions $[16,17]$. The treatment of choice for these enormous deeply located tumors which are 


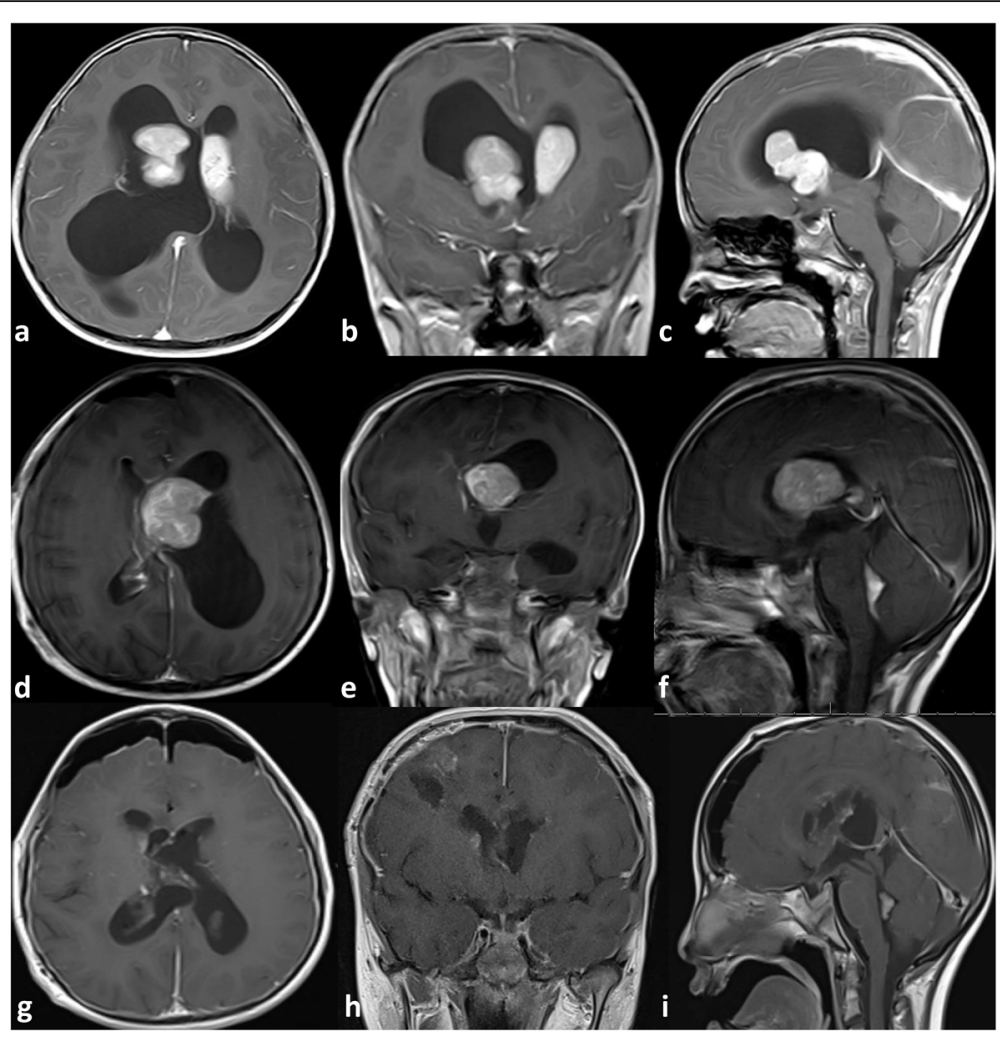

Fig. 2 Bilateral intraventricular sub-ependymal giant cell astrocytoma in an 8-year-old boy, who had generalized tonic-clonic convulsions. The patient had undergone an endoscopic biopsy and ventriculoperitoneal shunting, after which the seizures were controllable with medical treatment, and a decision of conservative management and serial follow-up imaging was taken. On 6-month follow-up, the seizures recurred and were difficult to control, and both tumors proved to have enlarged in size especially the right sided tumor. The right-sided tumor was surgically excised first through a right frontal trans-cortical approach, then the left-sided tumor was surgically excised in another session through a left frontal transcortical approach. a-c The initial axial, coronal, and sagittal contrast-enhanced brain MRI study. d-f Post-operative MRI following the first surgical session after excision of the right sided tumor which had enlarged during a 6-month follow-up period. $\mathbf{g}$-i The final postoperative axial, coronal, and sagittal contrast-enhanced brain MRI study, which demonstrates total excision of both tumors. No CSF diversion was needed in this patient

Table 2 Large or bilateral IVTs: primary main locations and extensions

\begin{tabular}{|c|c|c|c|c|c|c|}
\hline Primary main tumor location & Location pattern & & No. & $\%$ & Extension & No. \\
\hline \multirow[t]{2}{*}{ Frontal horn } & Frontal horn proper & 0 & 1 & $5 \%$ & Frontal horn & 7 \\
\hline & With extensions or bilaterality & 1 & & & & \\
\hline \multirow[t]{2}{*}{ Body } & Body proper & 5 & 11 & $55 \%$ & Body & 1 \\
\hline & With extensions or bilaterality & 6 & & & & \\
\hline \multirow[t]{2}{*}{ Temporal horn } & Temporal horn proper & 2 & 3 & $15 \%$ & Temporal horn & 0 \\
\hline & With extensions or bilaterality & 1 & & & & \\
\hline \multirow[t]{2}{*}{ Atrium } & Atrium proper & 2 & 5 & $25 \%$ & Atrium & 1 \\
\hline & With extensions or bilaterality & 3 & & & & \\
\hline \multirow[t]{2}{*}{ Occipital horn } & Occipital horn proper & 0 & 0 & $0 \%$ & Occipital horn & 3 \\
\hline & With extensions or bilaterality & 0 & & & & \\
\hline
\end{tabular}


Table 3 Summary of preoperative radiologic findings

\begin{tabular}{|c|c|c|}
\hline Item & Variable & No. \\
\hline \multirow[t]{5}{*}{ Primary anatomical tumor location } & Body & 11 \\
\hline & Atrium & 5 \\
\hline & Frontal horn & 1 \\
\hline & Occipital horn & 0 \\
\hline & Temporal horn & 3 \\
\hline \multirow[t]{3}{*}{ Maximal dimensions } & 3-4 cm (bilateral) & 1 \\
\hline & $5-7 \mathrm{~cm}$ & 8 \\
\hline & $7-9 \mathrm{~cm}$ & 11 \\
\hline \multirow[t]{2}{*}{ Preoperative hydrocephalus } & Present & 16 \\
\hline & Absent & 4 \\
\hline \multirow[t]{2}{*}{ Permanent postoperative CSF Diversion } & Needed & 9 \\
\hline & Not needed & 11 \\
\hline \multirow[t]{3}{*}{ T1 MRI } & Hyperintense & 4 \\
\hline & Isointense & 12 \\
\hline & Hypointense & 4 \\
\hline \multirow[t]{4}{*}{ Contrast enhancement } & Intensely enhanced & 9 \\
\hline & Moderately enhanced & 7 \\
\hline & Mildly enhanced & 3 \\
\hline & No enhancement & 1 \\
\hline \multirow[t]{3}{*}{ T2 MRI } & Hyperintense & 6 \\
\hline & Isointense & 13 \\
\hline & Hypointense & 1 \\
\hline \multirow[t]{2}{*}{ Peri-tumoral edema } & Present & 4 \\
\hline & Absent & 16 \\
\hline \multirow[t]{2}{*}{ Calcification } & Present & 6 \\
\hline & Absent & 14 \\
\hline
\end{tabular}

surrounded by eloquent normal brain is maximally safe surgical resection $[6,18-22]$.

We hereby report our results in the management of large intraventricular tumors through transcortical approaches in a series of 20 patients.

The main clinical presentations in the studied cases were manifestations of increased ICP in all cases, followed by visual deficits and seizures in 12 (60\%) and 7 (35\%) cases, respectively. Interestingly, there was no preoperative motor or sensory affection among our cases. On the other hand, Secer et al., reported preoperative headache in 23 (50\%), sensorial affection in 17 (36.95\%), motor affection in 16 (34.78\%), mental disorders in $5(10.87 \%)$, seizures in $4(8.70 \%)$, and visual affection in $2(4.35 \%)$ [17]. While D'angelo et al. reported headache in 13 cases (18.05\%), seizures in 12 cases (16.66\%), mental disorders in 21 cases (29.16), visual affection in 11 cases (15.27\%), and motor affection in 15 cases $(20.83 \%)$ [18].
The best surgical approach should give direct and clear access to the tumor and its blood supply through the shortest trajectory, while exerting minimal retraction forces and avoiding eloquent areas of the brain [23].

Piepmeier reported the commonest tumor locations to be the atrium, followed by the body and the frontal horns of the lateral ventricles [24], In the current study, the body of the lateral ventricle was considered the main tumor location in 11 cases, while 5 tumors arose from the atrium, 3 tumors arose from the temporal horns, and 1 case occupied mainly the frontal horn of the lateral ventricle.

Despite there is no clear definition as to delineate or to demarcate what could be considered a "large" intraventricular tumor, intraventricular tumors with maximal diameters of $5 \mathrm{~cm}$ and above usually constitute a significant challenge to the operating neurosurgeon, due to the deep location and the high vascularity of these tumors together with the potential need for brain retraction over 


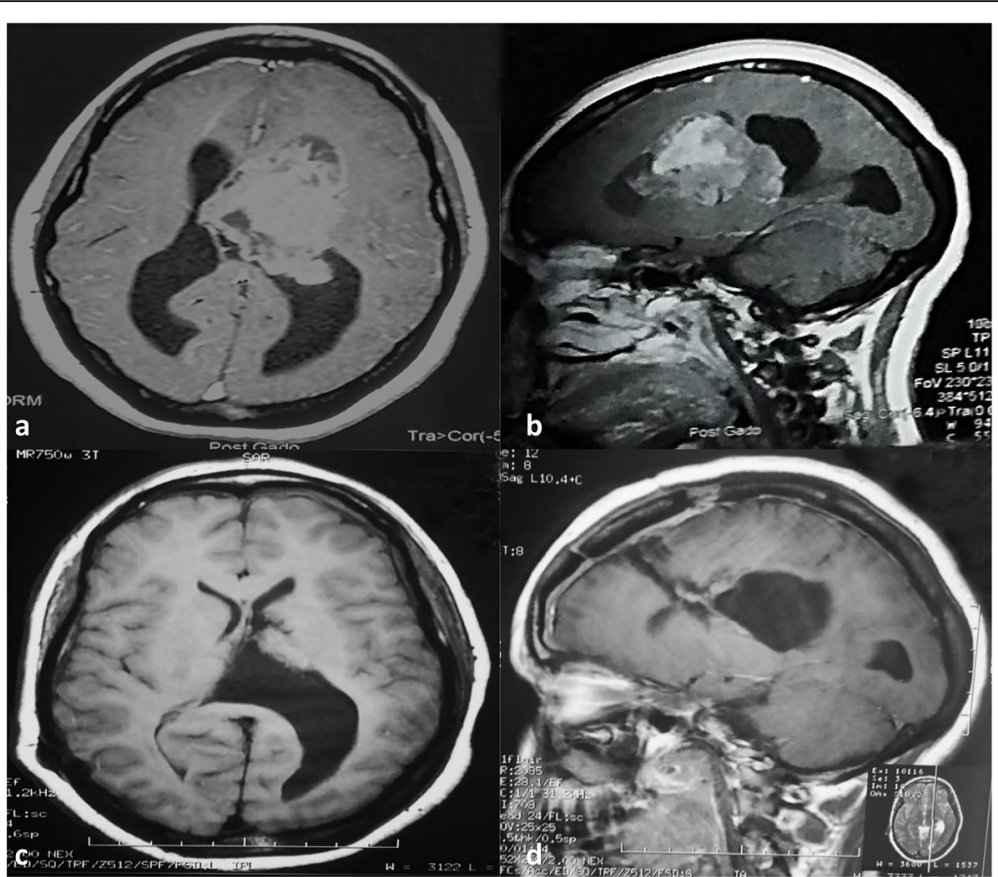

Fig. 3 A large intraventricular central neurocytoma in a 33-year-old lady. a, b The preoperative axial and sagittal contrast-enhanced brain MRI. c, d The postoperative axial and sagittal contrast-enhanced brain MRI study. Total excision of tumor was achieved following left frontal trans-cortical approach through the left middle frontal gyrus

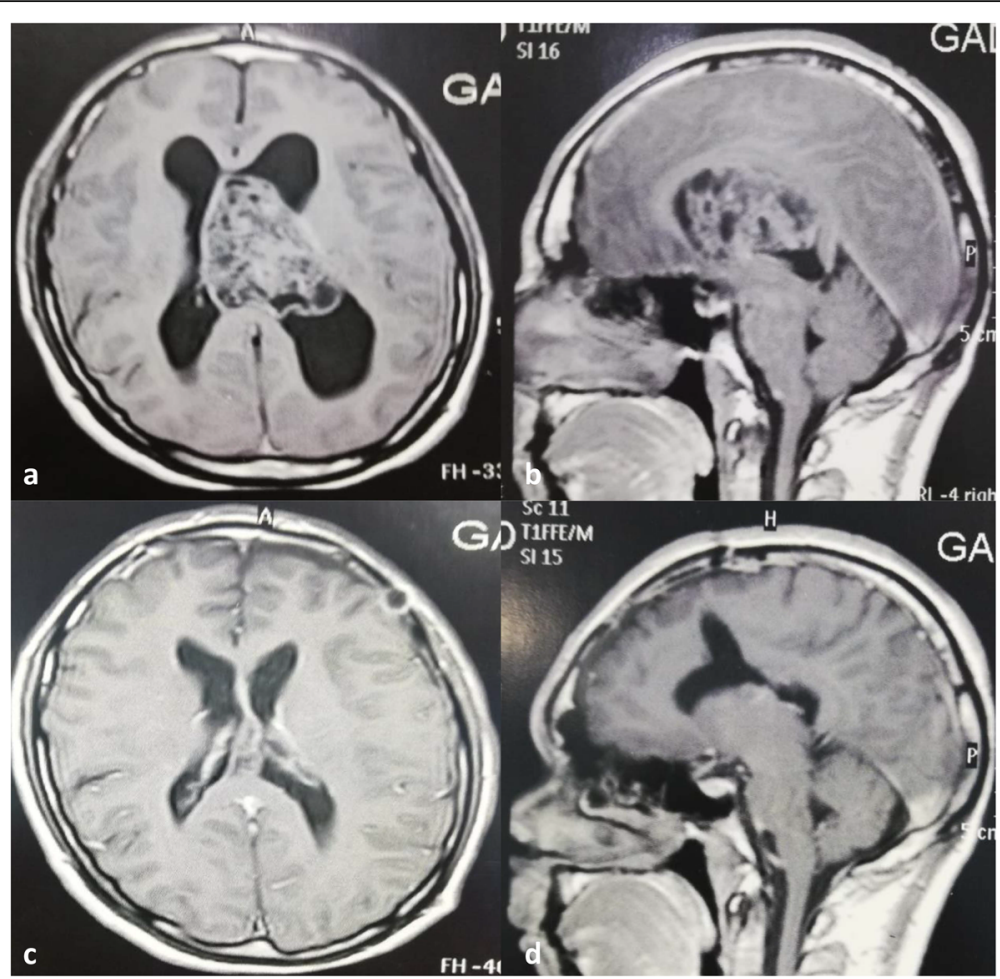

Fig. 4 A large intraventricular central neurocytoma in a 25-year-old male patient. a, b The preoperative axial and sagittal contrast-enhanced brain MRI study. $\mathbf{c}$, d The postoperative axial and sagittal contrast-enhanced brain MRI study. Total excision of tumor was achieved following left frontal trans-cortical approach through the left middle frontal gyrus 
Table 4 Histopathological diagnoses

\begin{tabular}{lll}
\hline Histopathological diagnosis & No. & $\%$ \\
\hline Central neurocytoma & 7 & $35 \%$ \\
Ependymoma & 3 & $15 \%$ \\
Subependymal giant cell astrocytoma & 3 & $15 \%$ \\
Choroid plexus carcinoma & 2 & $10 \%$ \\
Ganglioglioma & 2 & $10 \%$ \\
Atypical choroid plexus papilloma & 1 & $5 \%$ \\
Meningioma & 2 & $10 \%$ \\
\hline
\end{tabular}

or near eloquent brain regions. Therefore, postoperative neurological deficits are not uncommon in this context; and these may affect speech, motor, sensory and memory functions.

To the best of our knowledge, the literature lacks a clear definition of what tumor size is considered large enough to call for more precautions and approach and technique modification.

Considering the above-mentioned facts, the authors suggest a tumor size-based classification of intraventricular tumors; in which small tumors are those possessing less than $2.5 \mathrm{~cm}$ of maximum diameter, medium size tumors range from 2.5 to $5 \mathrm{~cm}$ in maximum diameters, and large intraventricular tumors possess more than 5 $\mathrm{cm}$ in maximum diameters. This classification would improve the appreciation and description of these challenging tumors and provides reasonable cut-off limits to define critical tumor sizes.

Accordingly, the maximum diameters of 19 of the 20 tumors presented in this article ranged from 5 to $9 \mathrm{~cm}$, while 1 tumor was $4 \mathrm{~cm}$ in diameter, and showed bilateral representation.

It was common for these tumors to have various anatomical extensions within the ventricular system (Table 2). But they shared a common criterion which is having a main area of involvement, usually the body (11

Table 5 Postoperative complications and morbidities

\begin{tabular}{lll}
\hline Postoperative complication/morbidity & No. & $\%$ \\
\hline Hydrocephalus & 9 & $45 \%$ \\
Minor intraventricular bleed & 9 & $45 \%$ \\
Subdural hygromas conservatively managed & 6 & $30 \%$ \\
Subdural hygromas mandated CSF shunt & 1 & $5 \%$ \\
Transient short-term memory affection & 4 & $20 \%$ \\
Transient mild motor deficits & 4 & $20 \%$ \\
Postoperative seizures & 2 & $10 \%$ \\
Moderate brain edema & 1 & $5 \%$ \\
Transient dysphasia & 1 & $5 \%$ \\
Extradural hematoma & 1 & $5 \%$ \\
\hline
\end{tabular}

CSF cerebrospinal fluid cases-55\%) and the atrium (5 cases-25\%). This concept was important in deciding which surgical approach to utilize to deal with these various extensions.

It was found that the frontal trans-cortical approach is a versatile approach which can be used to tackle tumors originating mainly within the ventricular body and the frontal horn. Similarly, the posterior parietal transcortical approach to the lateral ventricles was useful in tumors affecting mainly the atrium or the body of the lateral ventricles with occipital extensions (Fig. 5). Also, the middle temporal gyrus approach was useful in tackling IVTs which originated mainly in the temporal horns.

The transcortical approaches were utilized in all cases. Despite the large sizes of these tumors, gross total excision was achievable in 19 of these cases through transcortical approaches whether frontal, posterior parietal, or temporal. It is crucial to highlight the importance of avoiding much retraction over the cerebral cortex. Alternatively, proper patient positioning, medications, and anesthetic care usually provide adequate brain relaxation.

Significant intraoperative bleeding occurred mainly in six cases and was controllable by the usual techniques of hemostasis. There were no life-threatening intraoperative hemorrhages. The insertion of EVDs as a form of a temporary CSF diversion at the end of surgery was mainly the call of intraoperative judgement, which was decided based on the intraoperative impression of tumor vascularity, adequacy of hemostasis, and on the final evaluation of the surgical bed before closure.

In nine cases, there had been small postoperative intraventricular bleeds, which were managed by close observation for signs of increased ICP, along with serial CT brain scans as indicated. Ultimately, all these minor intraventricular bleeds were conservatively managed.

In the current study, permanent CSF diversion was resorted to in 9 cases (45\%); 8 of which had ventriculoperitoneal shunts and 1 had a subduro-peritoneal shunt. This goes in accordance with Danaila and others who concluded that abnormal postoperative CSF dynamics following tumor excision surgeries for IVTs mandate the insertion of ventricular shunts in a significant percentage of these patients ranging from 14.3 to $41.1 \%[16,25,26]$.

Regarding histopathological assessment, Gokalp et al. and others reported that the most common lateral ventricle tumors were ependymoma, astrocytoma, oligodendroglioma, choroid plexus papilloma, and meningioma $[6,18]$. Similarly, Secer et al. reported also that the most frequent tumor types were glioblastoma (19.57\%), choroid plexus papilloma (15.22\%), ependymoma (13.04\%), and meningioma (13.04\%) [17]. This, however, may also be affected by the mean ages of the studied patients. In the current study, the histopathological diagnoses (Table 4) included central neurocytoma in 7 cases (35\%) and 


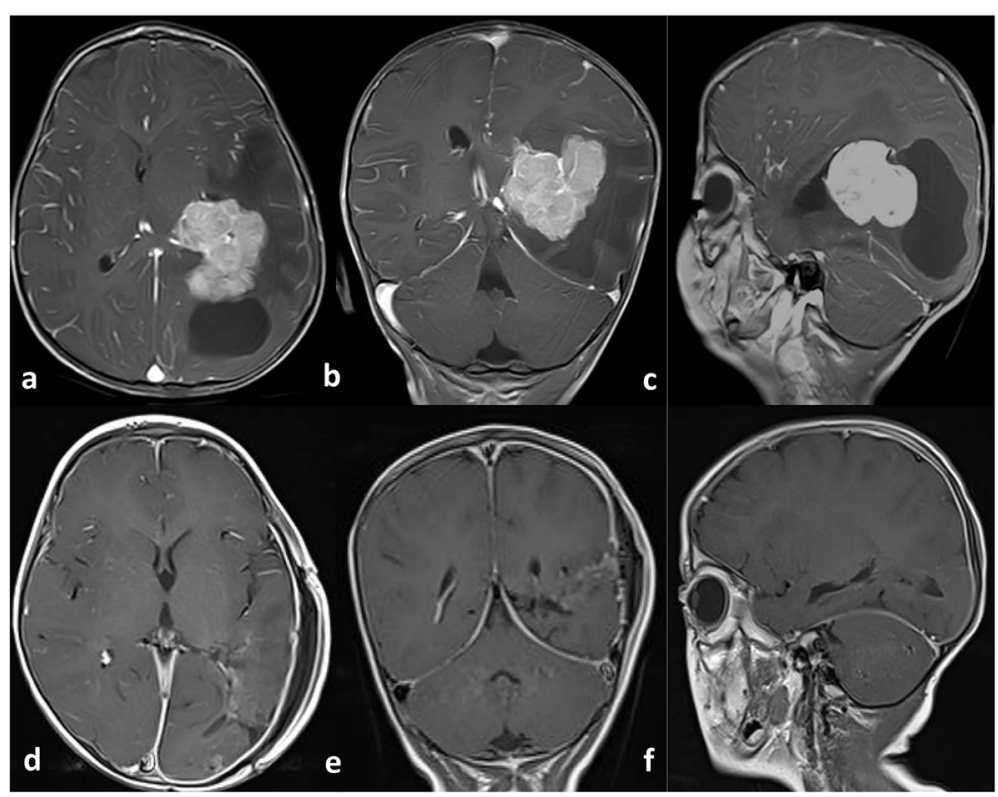

Fig. 5 A large left sided intraventricular choroid plexus carcinoma in a 3-year-old female patient. a-c The preoperative axial, coronal, and sagittal contrast-enhanced brain MRI study. d-f The postoperative axial, coronal, and sagittal contrast-enhanced brain MRI study. Total excision of the tumor was achieved following a left posterior parietal trans-cortical approach

ependymoma in 3 cases (15\%). Although there are various histopathological entities among IVTs, they all share common clinical and surgical challenges [2].

The morbidity rates of the operative routes to IVTs were reported to be up to $70 \%$, while the mortality rates range from 0 to $36 \%[18,20,21]$. In terms of surgical mortality, Pendl et al. and others, reported an operative mortality rate of $2.2-5 \%$. Causes included intracerebral hematomas and brain edema [3, 27]. On the other hand, no peri-operative mortalities were reported by Nishio et al., Sayyahmelli et al., and Lawton et al., as well [2830]. This goes hand in hand with the results of the current study which revealed no surgical mortalities.

The main postoperative neurological deficits encountered were transient short-term memory affection and transient motor weakness. All postoperative neurological deficits eventually improved within the first postoperative month. No permanent deficits occurred among the studied cases. Despite the trans-cortical approaches were utilized in all cases, postoperative seizures were only recorded in two cases, and these were short term and well-controlled. Moreover, the seven cases which had presented with preoperative seizures were successfully weaned from anti-convulsant medications within 3 months postoperatively. Postoperative complications or deficits were short term and reversible and did not affect the clinical outcomes of the studied cases. GOS was $5 / 5$ in all cases at 3, 6, and 9 months postoperatively. Table 4 shows a summary of postoperative morbidities.
The study limitations include the relatively small sample size, in addition to the relatively short follow-up period. Also, no specialized neuropsychological investigations were utilized in this study. The higher functions of the brain including memory testing were mainly assessed via thorough neurological examination, history taking, in addition to the GOS. Considering that postoperative deficits were mainly transient, which is quite unusual, using more specialized neuropsychological investigations could have probably helped detecting subtle permanent deficits in cognition, memory, or behavior. These limitations could be considered in our future research.

However, the excellent short-term clinical outcome results of our patients, along with the prospective nature of the study highlight that the transcortical trajectories to large or bilateral intraventricular tumors can be considered safe surgical options that give the shortest access to these challenging and rare tumors, provided that proper anesthetic management, patient positioning, and sound neurosurgical meticulous techniques are considered. In addition, a new tumor size-based classification of intraventricular tumors was proposed by the authors.

\section{Conclusion}

Surgical approaches to large tumors of lateral ventricles should be tailored to the variability of tumor locations, dimensions, extensions, and the individual morbid anatomy, in addition to the surgeon's experience and preferences. Trans-cortical approaches can provide safe 
surgical corridors to tackle these challenging subtypes of IVTs with good clinical outcomes, tumor resectability rates, and seizure control.

\section{Abbreviations}

CSF: Cerebrospinal fluid; CT: Computed tomography; EVDs: External ventricular drains; GOS: Glasgow Outcome Scale; ICP: Intracranial pressure; IVTs: Intraventricular tumors; MRI: Magnetic resonance imaging; REC: Research Ethics Committee; SEGA: Subependymal giant cell astrocytoma

\section{Acknowledgements}

The authors would like to acknowledge the studied patients and their families.

\section{Authors' contribution}

$M E, W E, W M$, and AF (all authors) performed the clinical part of the study and analyzed the data, in addition to writing, revising, and approving the manuscript. All authors have read and approved the final form of the manuscript.

\section{Funding}

This study had no funding from any resource.

\section{Availability of data and materials}

The data supporting our findings can be found with the corresponding author who can be contacted through the following email: dr.m. elkallaf@gmail.com

\section{Declarations}

\section{Ethics approval and consent to participate}

This study was conducted in Alexandria Main University Hospital following a study protocol that was approved by Research Ethics Committee of Faculty of Medicine under study No. 0201209, IRB No.: 00012098, and FWA No.: 00018699.

Written consent to participate was obtained from all patients or their nextof-kin before any intervention with explanation of all benefits and complication of any intervention.

The Ethics Committee of the Faculty of Medicine, Alexandria University is operated according to ICH GCP guidelines and applicable local and institutional regulations and guidelines which govern EC operation. Address: Faculty of Medicine, Alexandria University, 17 Champollion Street, El Messalah, Alexandria, Egypt. Email: alexmedethics@yahoo.com. Type of review: full board review.

Subject of research: human adult and pediatric males and females. Name, Email: Mohamed AbdAllah Elkallaf, dr.m.elkallaf@gmail.com. Name of the department: Department of Neurosurgery.

\section{Consent for publication}

Written consents for publication have been obtained from all patients or their next-of-kin and are available upon request.

\section{Competing interests}

The authors have no financial or non-financial conflicts to disclose.

Received: 25 January 2021 Accepted: 15 April 2021

Published online: 16 August 2021

\section{References}

1. Abbott KH, Courville CB. Intraventricular meningiomas: review of the literature and report of two cases. Bull Los Angeles Neurol Soc. 1942; 8:12-28.

2. De La Torre E, Alexander E Jr, Davis CH Jr, Crandell DL. Tumors of the lateral ventricles of the brain: Report of eight cases with suggestions of clinical management. J Neurosurg. 1963;20(6):461-70. https://doi.org/10.3171/jns.1 963.20.6.0461.

3. Pendl G, Oztürk E, Haselsberger K. Surgery of tumors of the lateral ventricle. Acta Neurochir (Wien). 1992;116(2-4):128-36. https://doi.org/1 $0.1007 / \mathrm{BF} 01540865$
4. Anderson RC, Ghatan S, Feldstein NA. Surgical approaches to tumors of the lateral ventricle. Neurosurg Clin N Am. 2003;14(4):509-25. https://doi.org/1 0.1016/S1042-3680(03)00054-8.

5. Delfini R, Acqui M, Oppido PA, Capone R, Santoro A, Ferrante L. Tumors of the lateral ventricles. Neurosurg Rev. 1991;14(2):127-33. https://doi.org/10.1 007/BF00313037.

6. Gokalp HZ, Yuceer N, Arasil E, Deda H, Attar A, Erdogan A, et al. Tumours of the lateral ventricle. A retrospective review of 112 cases operated upon 1970-1997. Neurosurg Rev. 1998;21 (2-3):126-37. https://doi.org/10.1007/ BF02389318.

7. Purves D, Augustine GJ, Fitzpatrick D, et al., editors. Neuroscience. 2nd edition. Sunderland (MA): Sinauer Associates; 2001. The Ventricular System. Available from: https://www.ncbi.nIm.nih.gov/books/NBK11083/. Accessed 10 Dec 2020.

8. Shenoy SS, Lui F. Neuroanatomy, Ventricular System. [Updated 2020 Jul 31]. In: StatPearls. Treasure Island (FL): StatPearls Publishing; 2021 Jan-. Available from: https://www.ncbi.nlm.nih.gov/books/NBK532932/. Accessed 10 Dec 2020.

9. Elwatidy SM, Albakr AA, Al Towim AA, Malik SH. Tumors of the lateral and third ventricle: surgical management and outcome analysis in 42 cases. Neurosciences (Riyadh). 2017;22(4):274-81. https://doi.org/10.17712/nsj.201 7.4.20170149.

10. Xie T, Zhou L, Zhang $X$, Sun W, Ding H, Liu T, et al. Endoscopic supracerebellar transtentorial approach to atrium of lateral ventricle: preliminary surgical and optical considerations. World Neurosurg. 2017;105: 805-11. https://doi.org/10.1016/j.wneu.2017.06.093.

11. Abramov I, Zhao X, Belykh E, Lawton MT, Pitskhelauri D, Preul MC. Supracerebellar infratentorial inverted subchoroidal approach to lateral ventricle lesions: Anatomical study and illustrative case. Surg Neurol Int. 2021;3(12):39.

12. Hendrix $\mathrm{P}$, Senger $\mathrm{S}$, Griessenauer $\mathrm{CJ}$, Simgen A, Linsler $\mathrm{S}$, Oertel J. Preoperative navigated transcranial magnetic stimulation and tractography in transparietal approach to the trigone of the lateral ventricle. J Clin Neurosci. 2017;41:154-61. https://doi.org/10.1016/j.jocn.2017.02.029.

13. Nakajo K, Uda T, Goto T, Morisako H, Nishijima S, Kawashima T, et al. Changes in cognitive function after resection of lesions in the anterior part of the lateral ventricle via an interhemispheric transcallosal approach. J Clin Neurosci. 2020;79:39-44. https://doi.org/10.1016/j.jocn.2020.07.026.

14. Oliveira Ramalho C, Viguini Tolentino Correa A, Hilton Vieira Madeira T, Nascimento Ottoni A, Tau Zymberg S. Unilateral hydrocephalus due to lateral ventricle colloid cyst treated by neuroendoscopic approach. Cureus. 2020;12(4):e7825.

15. Xing H, Jing-Ping L, Kun-Ming Q, Shen-Chao L, Chun-Hai T, Jing-Zhan W. Transcallosal-lateral ventricle-choroid fissure approach for excising large pineal region tumors extending into the third ventricle: experience in 15 pediatric cases. Childs Nerv Syst. 2021;37(2):671-6. https://doi.org/10.1007/ s00381-020-04742-z.

16. Dănăilă L. Primary tumors of the lateral ventricles of the brain. Chirurgia (Bucur), 2013:108(5):616-30.

17. Seçer HI, Düz B, Izci Y, Tehli O, Solmaz I, Gönül E. Tumors of the lateral ventricle: the factors that affected the preference of the surgical approach in 46 patiens. Turk Neurosurg. 2008;18(4):345-55.

18. D'Angelo VA, Galarza M, Catapano D, Monte V, Bisceglia M, Carosi I. Lateral ventricle tumors: surgical strategies according to tumor origin and development--a series of 72 cases. Neurosurgery. 2005;56:36-45 discussion 36-45.

19. Darkwah Oppong M, Muller O, Jabbarli R, Dammann P, Sure U, El Hindy N. Intraventricular mass lesions: Still a question of surgical approach? J Clin Neurosci. 2017;43:157-62. https://doi.org/10.1016/j. jocn.2017.05.036

20. Hassaneen W, Suki D, Salaskar AL, Levine NB, DeMonte F, Lang FF, et al. Immediate morbidity and mortality associated with transcallosal resection of tumors of the third ventricle. J Clin Neurosci. 2010;17(7):830-6. https://doi. org/10.1016/j.jocn.2009.12.007.

21. Milligan BD, Meyer FB. Morbidity of transcallosal and transcortical approaches to lesions in and around the lateral and third ventricles: a single-institution experience. Neurosurgery. 2010;67(6):1483-96; discussion 96. https://doi.org/10.1227/NEU.0b013e3181f7eb68.

22. Zuccaro G, Sosa F, Cuccia V, Lubieniecky F, Monges J. Lateral ventricle tumors in children: a series of 54 cases. Childs Nerv Syst. 1999;15(11-12): 774-85. https://doi.org/10.1007/s003810050470. 
23. Tew JM, Lewis Al, Reichert KW. Management strategies and surgical techniques for deep-seated supratentorial arteriovenous malformations. Neurosurgery. 1995;36(6):1065-72. https://doi.org/10.1227/00006123-1 99506000-00001.

24. Piepmeier JM. Tumors and approaches to the lateral ventricles. Introduction and overview. J Neurooncol. 1996;30(3):267-74. https://doi.org/10.1007/ BF00177278.

25. Piepmeier JM, Sass KJ. Surgical management of lateral ventricular tumors. In: Paoletti P, Takakura K, Walker M, editors. Neuro-Oncology. Dordecht: Kluwer Academic Publishers; 1991. p. 333-5. https://doi.org/10.1007/978-94-011-31 52-0_59.

26. Mazher S, Imran M, Ashraf J, Ahmed A, Shah IU, Zulfiqar F. Outcome of open transcortical approach in the management of intraventricular lesions. J Coll Physicians Surg Pak. 2013;23(12):857-61.

27. Aftahy AK, Barz M, Krauss P, Liesche F, Wiestler B, Combs SE, et al. Intraventricular neuroepithelial tumors: surgical outcome, technical considerations and review of literature. BMC Cancer. 2020;20(1):1060.

28. Nishio S, Fujiwara S, Tashima T, Takeshita I, Fujii K, Fukui M. Tumors of the lateral ventricular wall, especially the septum pellucidum: clinical presentation and variations in pathological features. Neurosurgery. 1990; 27(2):224-30. https://doi.org/10.1227/00006123-199008000-00009.

29. Lawton MT, Golfinos JG, Spetzler RF. The contralateral transcallosal approach: experience with 32 patients. Neurosurgery. 1996;39(4):729-34. https://doi.org/10.1097/00006123-199610000-00016.

30. Sayyahmelli S, Baran O, Uğurlar D, Kemerdere R, Antar V, Tanriverdi T. Intracranial intraventricular tumors: long-term surgical outcome of 25 patients. Turk J Med Sci. 2017:47(1):76-84.

\section{Publisher's Note}

Springer Nature remains neutral with regard to jurisdictional claims in published maps and institutional affiliations.

\section{Submit your manuscript to a SpringerOpen ${ }^{\circ}$ journal and benefit from:}

- Convenient online submission

- Rigorous peer review

- Open access: articles freely available online

- High visibility within the field

- Retaining the copyright to your article

Submit your next manuscript at $\boldsymbol{\nabla}$ springeropen.com 